\title{
Gas Sensing and Power Harvesting Polyvinylidene Fluoride Nanocomposites Containing Hybrid Nanotubes
}

\author{
DEEPALEKSHMI PONNAMMA $\odot,{ }^{1,4}$ ASHOK K. SHARMA, ${ }^{2}$ \\ PRIYA SAHARAN, ${ }^{2}$ and MARIAM AL ALI AL-MAADEED ${ }^{1,3}$ \\ 1.-Center for Advanced Materials, Qatar University, P O Box 2713, Doha, Qatar. \\ 2.-Department of Materials Science and Nanotechnology, Deenbandhu Chhotu Ram University \\ of Science and Technology, Murthal, Haryana 131001, India. 3.-Materials Science and \\ Technology Program (MATS), College of Arts and Sciences, Qatar University, P O Box 2713, \\ Doha, Qatar. 4.—e-mail: lekshmi_deepa@yahoo.com
}

Gas sensing properties at room temperature and energy harvesting performances are realized for the polyvinylidene fluoride (PVDF) nanocomposites containing titanium dioxide $\left(\mathrm{TiO}_{2}\right)$ nanotubes grown in the presence of carbon nanotubes (CNT). While hydrothermal reaction is practiced for the development of $\mathrm{TiO}_{2} / \mathrm{CNT}$ hybrid nanotubes, spin coating is done for the nanocomposite films to be deposited on sensing electrodes. Influence of various filler concentrations and the synergistic combination of fillers on the sensing characteristics are studied by recording the response times and the stability of the results. Upon exposure to liquefied petroleum gas, the $\mathrm{PVDF} / \mathrm{TiO}_{2}-\mathrm{CNT}$ (2.5 wt.\%) gas sensor shows a sensing response of $0.45 \mathrm{~s}$ (400 ppm LPG), approximately nine times higher than the composite containing $2.5 \mathrm{wt} . \%$ of $\mathrm{TiO}_{2}$ or 2.5 wt.\% CNT. The piezoelectric response of the samples is also recorded and correlated with the synergistic influence of the filler materials. The current study can stimulate a good trend in fabricating self-powered gas sensors from PVDF nanocomposites.

Key words: Filler synergy, gas sensing, nanocomposite, nanogenerator, solvent vapors

\section{INTRODUCTION}

Advancement of flexible and conductive polymer nanocomposites requires the presence of a highly conducting network arranged through electrically conducting filler units. ${ }^{1}$ Carbon based fillers including carbon black, carbon fibers, carbon nanotubes (CNT) and graphene can improve electrical conductivity of various polymer nanocomposites in addition to mechanical stability. ${ }^{2,3}$ The intrinsic conductivity of such fillers enable their nanocomposites for multifunctional applications in sensing, actuators, biomedical devices for health monitoring, etc., ${ }^{4,5}$ Sensing depends on the variation in electrical resistance when the nanocomposite is exposed to

(Received September 29, 2019; accepted December 17, 2019; published online January 3, 2020) external stimulus such as temperature ${ }^{6}$ organic solvent, ${ }^{7}$ pressure, ${ }^{8}$ strain ${ }^{9}$ and the solvent vapors/ gas. ${ }^{10}$ In the polymer nanocomposites containing conductive filler particles, conductive pathways are generated by direct contact between the nanomaterials and by tunneling/electron hopping effects. This mainly happens when the concentration of the nanomaterials becomes well above the percolation threshold. ${ }^{11}$

Single and multi-walled carbon nanotubes (SWCNT and MWCNT) are promising nanomaterials for improving the electrical and mechanical properties of polymers, and many sensors are reported based on these materials. ${ }^{12}$ However, the overall conductivity of the polymer/CNT nanocomposite depends on the filler concentration, nature of connecting networks in terms of dispersion and orientation of nanotubes and the CNT-polymer interfacial interactions. This is often achieved by 
surface modification or compatibilization techniques including chemical functionalization of nanotubes. ${ }^{13,14}$ Wang et al. applied the principle of $3 \mathrm{D}$ self-segregated structures for fabricating polydimethylsiloxane/MWCNT nanocomposite with high electrical conductivity of $0.003 \mathrm{~S} \mathrm{~m}^{-1}$, at 0.2 vol.\% of MWCNT, maximizing the interfacial interaction through the formation of chemical bonds. ${ }^{15}$ Hybrid nanocomposites are other alternatives to achieve maximum properties with excellent filler dispersion due to the synergistic influences of the fillers. Hosseini and Yousefi ${ }^{16}$ proposed a piezoelectric sensor from the electrospun fibers of polyvinylidene fluoride (PVDF) containing different ratios of MWCNT and Cloisite 30B. While the clay nanolayers enhanced the beta phase content of the PVDF, and thus the piezoelectric properties, the MWCNT decreased the impedance and increased the dielectric constant of the composite. CNT decorated with gold nanoparticles with a unique 1D/0D structure was developed on a commercial PVDF membrane targeting an application of a surface enhanced Raman spectroscopy sensor. ${ }^{17}$ Those plasmonic nanostructures maintained good uniformity in dispersion and were capable of detecting various molecular contaminants in food and aqueous samples. This hybrid architecture for the nanoparticles can enhance the characteristic properties because of the synergistic effect of material behavior and by the uniform distribution of each of them through network formation.

PVDF is an electroactive polymer with stretchable design, good biocompatibility and low acoustic impedance so that many sensors and lightweight energy harvesting devices are made of this polymer based nanocomposites. ${ }^{16,18-22}$ Since the beta phase of the PVDF contributes to its polarizability and piezoelectricity, the alpha to beta or gamma to beta phase conversion is highly anticipated while incorporating various fillers into it. PVDF based flexible sensors are attaining much significance as piezoelectric nanogenerators, sound absorbers and typical sensors. ${ }^{16}$ Since PVDF is thermally, chemically and optically stable to radiation exposures, structural health monitoring applications of PVDF based materials in civil infrastructures and airplanes are significant. ${ }^{11}$ Georgousis et al. reported the electrical resistance variation of PVDF composites upon the incorporation of different concentrations of MWCNTs. ${ }^{2}$ The authors noticed that a higher percolation threshold of $8 \mathrm{wt}$.\% is more suitable for those applications demanding high deformation or stress whereas a lower concentration limit of $1.5 \mathrm{wt} . \%$ is suitable for measuring lower strains. The whole study focused on the impact of CNT concentrations on the nanocomposite sensing and the formation and breaking of filler networks. The ability of better conducting network formation of the CNTs, when compared to reduced graphene oxide, was also reported by $\mathrm{Hu}$ et al. by the fabricated polylactic acid nanocomposite strain sensors. ${ }^{23}$ This is because of the better rearrangement of CNT nanotubes when compared to the slippage observed in reduced graphene oxide layers. Since powergenerating sensors are applicable in remote sensing, autonomous and wireless sensors, detailed investigation of their fabrication has the most significance.

Our group has investigated the piezoelectric and sensing properties of the PVDF composites in the presence of semiconducting and carbonaceous fillers. ${ }^{8,18-21}$ In addition, the solvent sensing characteristics of PVDF nanocomposites containing a hybrid combination of titanium dioxide $\left(\mathrm{TiO}_{2}\right)$ nanotubes and MWCNTs were addressed in our study. ${ }^{24}$ The present paper is a subsequent study of sensing characteristics of the same $\mathrm{PVDF} / \mathrm{TiO}_{2^{-}}$ MWCNT composites towards the liquefied petroleum gas (LPG). Synergistic influence of the filler combination on the electrical and sensing properties of piezoelectric PVDF through better interfacial interactions and crystallinity enhancement is the main motive behind the research. The $\mathrm{TiO}_{2}$ semiconducting nanotubes are grown in the presence of CNTs by the hydrothermal method, and later added to the PVDF matrix by solution casting. The sensing and piezoelectric energy harvesting properties of the composites are correlated with the network formation and percolation threshold values and the LPG sensing profiles are recorded with and without thermal treatment. The responses of the sensor towards different solvent vapors and its self-powering capacity by means of piezoelectric output voltage are investigated. The dielectric properties are also studied to explain the energy storage performance of the samples. A good correlation is drawn between the presence of piezoelectric crystalline phase formation in PVDF, the synergistic influence of hybrid filler nanotubes, and the sensing responses towards the various gases.

\section{EXPERIMENTAL TECHNIQUES}

\section{Materials}

PVDF pellets of molecular weight $M_{\mathrm{w}}$ $1,80,000 \mathrm{~g} / \mathrm{mol}, \mathrm{TiO}_{2}$ anatase powder and the MWCNT were commercially obtained from Sigma Aldrich. Solvents, acetone and dimethylformamide (DMF) and sodium hydroxide $(\mathrm{NaOH})$ were purchased from British drug house (BDH) chemicals, Doha.

\section{Synthesis of $\mathrm{TiO}_{2}$ Nanotubes and CNT-TiO Hybrid Nanotubes}

$\mathrm{TiO}_{2}$ nanotubes were synthesized by the hydrothermal method. ${ }^{25}$ For this specific quantity $(2.4 \mathrm{~g})$ of $\mathrm{TiO}_{2}$ anatase micropowder was mixed with $10 \mathrm{~N} \mathrm{NaOH}(40 \mathrm{ml})$ by continuously stirring for 15 min and then transferred to an autoclave kept at $130^{\circ} \mathrm{C}$, and the heating was continued for $10 \mathrm{~h}$. The same procedure was done in the presence of $0.01 \mathrm{~g}$ 
carbon nanotube $(\mathrm{CNT})$ so that $\mathrm{TiO}_{2}$ nanotubes are grown on it. ${ }^{24}$

\section{Synthesis of PVDF Nanocomposites}

Solution casting method was employed for fabricating the PVDF nanocomposites containing CNT and $\mathrm{TiO}_{2}$ nanotubes. For this, specific amounts $(2 \mathrm{~g}$ each) of PVDF pellets were magnetically stirred at $70^{\circ} \mathrm{C}$ for $2-3 \mathrm{~h}$ in DMF/Acetone $1: 1$ solvent mixture. The CNT, $\mathrm{TiO}_{2}$ nanotubes and $\mathrm{CNT}-\mathrm{TiO}_{2}$ nanohybrids in particular weight percentages (1 wt.\%, 2.5 wt.\% and 5 wt.\%) were separately dispersed in the same solvent mixture by ultrasonication and added to the PVDF dissolution by room temperature magnetic stirring overnight. Final composite films were obtained by drying the dispersions in an oven and hot pressing at $170^{\circ} \mathrm{C}$. There were six sets of samples for the analysis as represented below.

Neat PVDF (Neat), PVDF with 1 wt. $\% \mathrm{CNT}^{-\mathrm{TiO}_{2}}$ hybrid nanomaterial (PCT1), PVDF with 2.5 wt.\% CNT-TiO ${ }_{2}$ hybrid nanomaterial (PCT2.5), PVDF with 5 wt.\% $\quad \mathrm{CNT}^{-\mathrm{TiO}_{2}}$ hybrid nanomaterial (PCT5), PVDF with 2.5 wt.\% CNT (PC), PVDF with 2.5 wt.\% $\mathrm{TiO}_{2}(\mathrm{PT})$.

\section{Characterization Methods}

Sensing experiments were done using specially designed chambers. ${ }^{26,27}$ Prior to the experiments, samples were coated on pre-patterned interdigitated electrodes (Fig. 1) by drop drying process. These coated electrodes were dried at $40^{\circ} \mathrm{C}$ for $1 \mathrm{~h}$ in an oven. For sensing, an in-house gas sensor chamber was made with specific inlet and outlet vents for the test gases to enter and exit. The resistance changes when analyte gases contact the samples, and these variations were monitored using the Keithley multimeter (Model 2750), connected to a computer. The exit vent was connected to a suction pump to remove the gases and the responses (sensitivity) were calculated by the following Eq. 1.

$$
S=\frac{R_{\mathrm{a}}-R_{\mathrm{g}}}{R_{\mathrm{a}}},
$$

where $R_{\mathrm{a}}$ and $R_{\mathrm{g}}$ represent the resistance values in air and gas, respectively.

Piezoelectric characteristics of the samples were recorded using the laboratory set up consisting of a frequency generator, amplifier, vibrating shaker and data acquisition unit. ${ }^{28}$ While the shaker vibrates according to the amplified generated frequency $(15-50 \mathrm{~Hz})$, the specific weights $(2.5 \mathrm{~N}) \mathrm{kept}$ on the sample surface imparted compressive force on the sample. These vibrational forces created dipole moment variations within the samples and generated piezoelectric voltage signals which were obtained from a data acquisition software. For the piezoelectric energy harvesting experiments, the samples were made by electrically poling the solvent casted film of $0.2 \mathrm{~mm}$ thickness at $8 \mathrm{kV}$ for $7 \mathrm{s.}{ }^{18,22}$

Sample morphology was analyzed by scanning electron microscopy (SEM) and transmission electron microscopy (TEM). The SEM studies were conducted by (SEM, XL-30E Philips Co., Holland), TEM by a Transmission Electron Microscope FEI TECNAI G ${ }^{2}$. FTIR spectra of the samples were with a PerkinElmer Spectrum 400 spectrophotometer in the range $400-4000 \mathrm{~cm}^{-1}$ with a resolution of $2 \mathrm{~cm}^{-1}$. X-ray diffraction studies were performed by XRD diffractometer (Mini Flex 2, Rigaku). Nickel filtered $\mathrm{CuK} \alpha$ radiation $(\lambda=0.1564 \mathrm{~nm})$ operated at $30 \mathrm{~V}$ and $15 \mathrm{~mA}$ served as the source. The patterns were recorded in the $2 \theta$ range of $5^{\circ}-80^{\circ}$ at a scanning speed of $1.8 \% \mathrm{~min}$. Broadband dielectric/ impedance spectroscopy-Novocontrol was used for

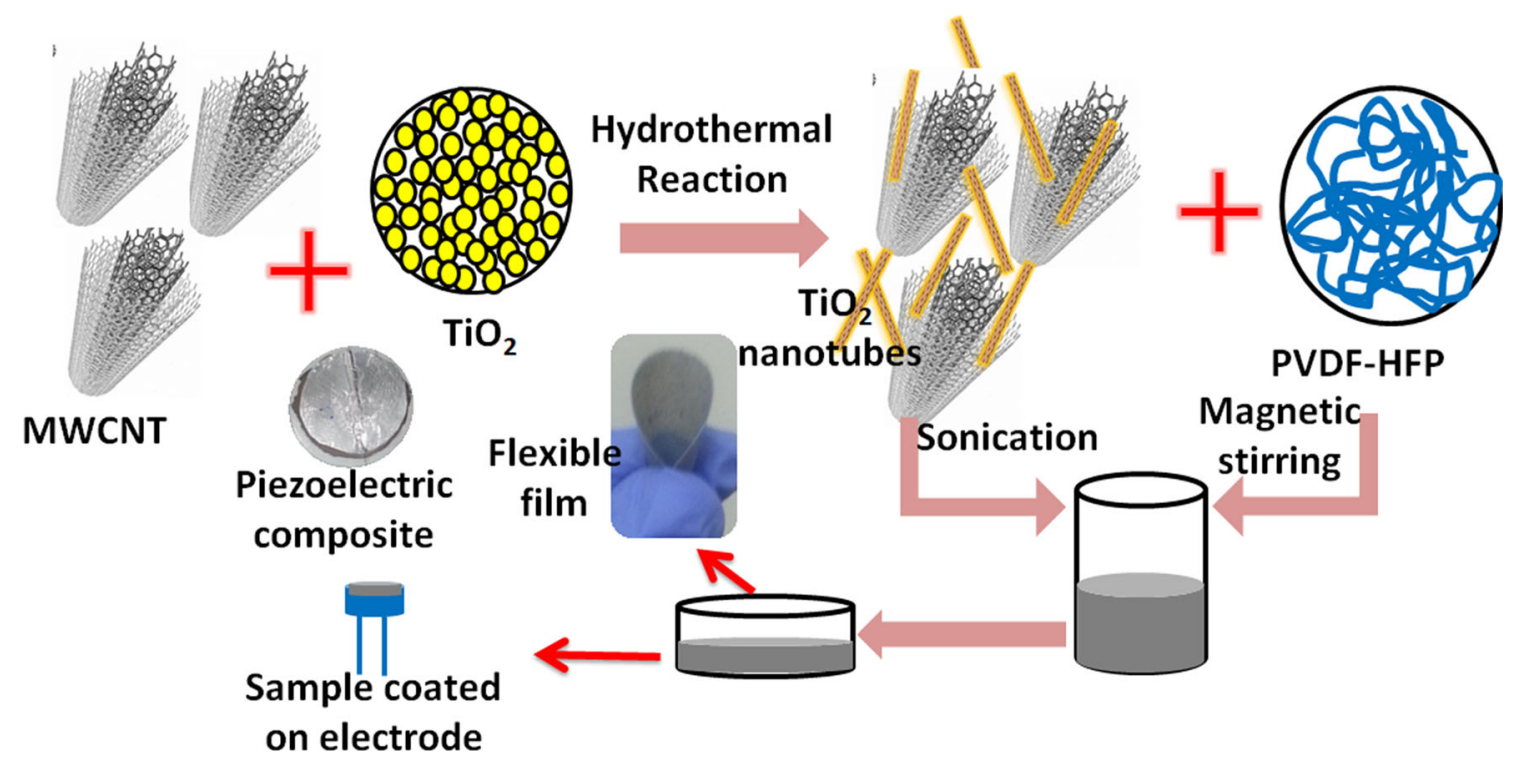

Fig. 1. Schematic representation of the sample preparation. 
measuring the dielectric properties of the samples. The dielectric constant, dielectric loss, $\tan \delta$ and conductivity values of the samples were checked during the frequency range of $10^{-2} \mathrm{~Hz}$ to $10^{6} \mathrm{~Hz}$.

\section{RESULTS AND DISCUSSION}

Figure 1 outlines the preparation processes involved in the PVDF nanocomposite film development. The $\mathrm{TiO}_{2}$ powders are spherical shaped and the hydrothermal reaction ruptures the spherical morphology transforming them to layers and thereafter tubes. The $\mathrm{TiO}_{2}$ nanotubes developed in the presence of MWCNTs form a hybrid structural pattern, where the nanotubes are segregated and well distributed. ${ }^{24}$ After purifying the hybrid powder, it was dispersed in DMF: Acetone solvent mixture and finally mixed with PVDF. Later it was magnetically stirred and developed to flexible nanosheets as indicated in Fig. 1. The coated samples on the sensing electrodes are also shown in the figure. Such electrodes with coated samples were used for the LPG and solvent vapor sensing experiments.

The sensing characteristics of the samples towards LPG from $100 \mathrm{ppm}$ to $700 \mathrm{ppm}$ concentration are plotted in Fig. 2. All polymer nanocomposite samples are not analyzed because of the nonconducting (very high resistance) behavior of certain samples. This includes the neat polymer without any conductive filler particles embedded in it and the PCT1 sample containing conductive filler particles below the percolation threshold. ${ }^{29}$ All other samples-PCT2.5, PCT5, PT and PC-showed increasing sensor responses with respect to LPG concentration (Fig. 2a). However the response is quite high for the sample PCT2.5 containing the hybrid $\mathrm{CNT}^{-\mathrm{TiO}_{2}}$ nanotubes in 2.5 wt.\%. This is because, at the $2.5 \mathrm{wt} \%$ filler amount, the concentration is near to/above the percolation threshold value, and this initiated good network formation among the nanotubes. ${ }^{30}$ However the $\mathrm{PC}$ and PT samples, which contain $\mathrm{CNT}$ and $\mathrm{TiO}_{2}$ nanotubes in 2.5 wt.\%, showed lower sensing responses. This result shows that the hybrid nanostructure enhanced the individual nanotube dispersability within the PVDF chains. ${ }^{31}$

Filler synergy enhanced sensing capacity observed in this report is comparable with the LPG sensing activity for the ZnO-MWCNT nanocomposite. ${ }^{32}$ Maximum average sensitivity of 41.95 for $1500 \mathrm{ppm}$ LPG gas with a sensing response of $61.57 \mathrm{~s}$ was observed in their report, and our results show better response time $(0.45 \mathrm{~s})$ at lower LPG concentration (400 ppm). In addition, the widely reported polyaniline composites also give comparatively lower sensing response, at higher filler concentrations. For instance the $\mathrm{Fe}_{2} \mathrm{O}_{3} /$ polyaniline composites report response time of $60 \mathrm{~s}$ for $200 \mathrm{ppm}$ LPG at $3 \mathrm{wt} . \%$ composition ${ }^{33}$ and the $\mathrm{TiO}_{2} /$ polyaniline composite reports $90 \%$ sensing response at $30 \mathrm{wt} . \%$ composition (400 ppm LPG). ${ }^{34}$ These results clearly show the significance of flexible sensing devices at lower filler concentration and with better response time.

Sensing responses by different PVDF composites depend on various factors. During LPG exposure electron transfer reactions occur within the samples, these redox reactions have positive influence in causing resistance variations. In addition, the sample surface acts as adsorption sites for gas molecules by replacing the atmospheric oxygen. When the sample is exposed to LPG, the oxygen present in the surface sites react with the gas molecules through various intermediate reaction steps. ${ }^{10,26}$

$$
\begin{gathered}
\mathrm{C}_{n} \mathrm{H}_{2 n+2}+2 \mathrm{O}^{-} \rightarrow \mathrm{C}_{n} \mathrm{H}_{2 n}: \mathrm{O}+\mathrm{e}^{-}+\mathrm{H}_{2} \mathrm{O} \\
\mathrm{C}_{n} \mathrm{H}_{2 n}: \mathrm{O}+\mathrm{O}^{-} \rightarrow \mathrm{CO}_{2}+\mathrm{H}_{2} \mathrm{O}+\mathrm{e}^{-},
\end{gathered}
$$

where the $\mathrm{C}_{n} \mathrm{H}_{2 n+2}$ represents the alkanes such as $\mathrm{C}_{4} \mathrm{H}_{10}, \mathrm{C}_{3} \mathrm{H}_{8}$ and $\mathrm{CH}_{4}$ and $\mathrm{C}_{\mathrm{n}} \mathrm{H}_{2 \mathrm{n}}$ : $\mathrm{O}$ represents the partially oxidized reaction intermediate on the sample surface. It is clear from Fig. $2 b$ that concentration of LPG enhances the sensing responses of the composite samples. At a particular temperature of $50^{\circ} \mathrm{C}$, all samples showed pronounced influence on sensing the LPG analyte molecules. However, the PCT2.5 sample showed the consistent result. Figure 2c represents the variation in sensing characteristics with temperature at a particular concentration of LPG (400 ppm). ${ }^{27}$ For PT, PC and PCT5 samples, the increase in sensing response is noticed with temperature that can be due to the reduction in adsorbed water molecules causing increased resistance. $^{26}$ Good temperature sensitivity is observed for the PCT2.5 sample, in which the $\mathrm{CNT}^{-\mathrm{TiO}_{2}}$ hybrid nanotubes are present at $2.5 \mathrm{wt} . \%$.

Resistance variations for PCT2.5 to different LPG concentrations at room temperature is depicted in Fig. 3a. A gradual increase in resistance was observed for the nanocomposite when the LPG concentration was changed from $100 \mathrm{ppm}$ to $700 \mathrm{ppm}$. The recovery of this particular nanocomposite was found to be good as it returns to its original stage quickly on exposure to air. ${ }^{30}$ The resistance variation of PCT2.5 according to the temperature differences is represented in Fig. 3b. Though large variations in resistance was seen at lower temperatures, higher temperatures above $50^{\circ} \mathrm{C}$ imbalanced the resistance variations. This might be due to the higher activation energy of gas molecules and the higher rate of electro transfer reactions. ${ }^{35}$ It can be correlated with the synergistic filler effect and the better network formation of both fillers.

The sensing responses of the sample towards different solvent vapors were also studied to test the selective performance. ${ }^{19}$ Figure $4 \mathrm{a}$ shows the relative resistance variations of the PVDF-2.5CTNT sample towards the vapors of benzene, acetone, 

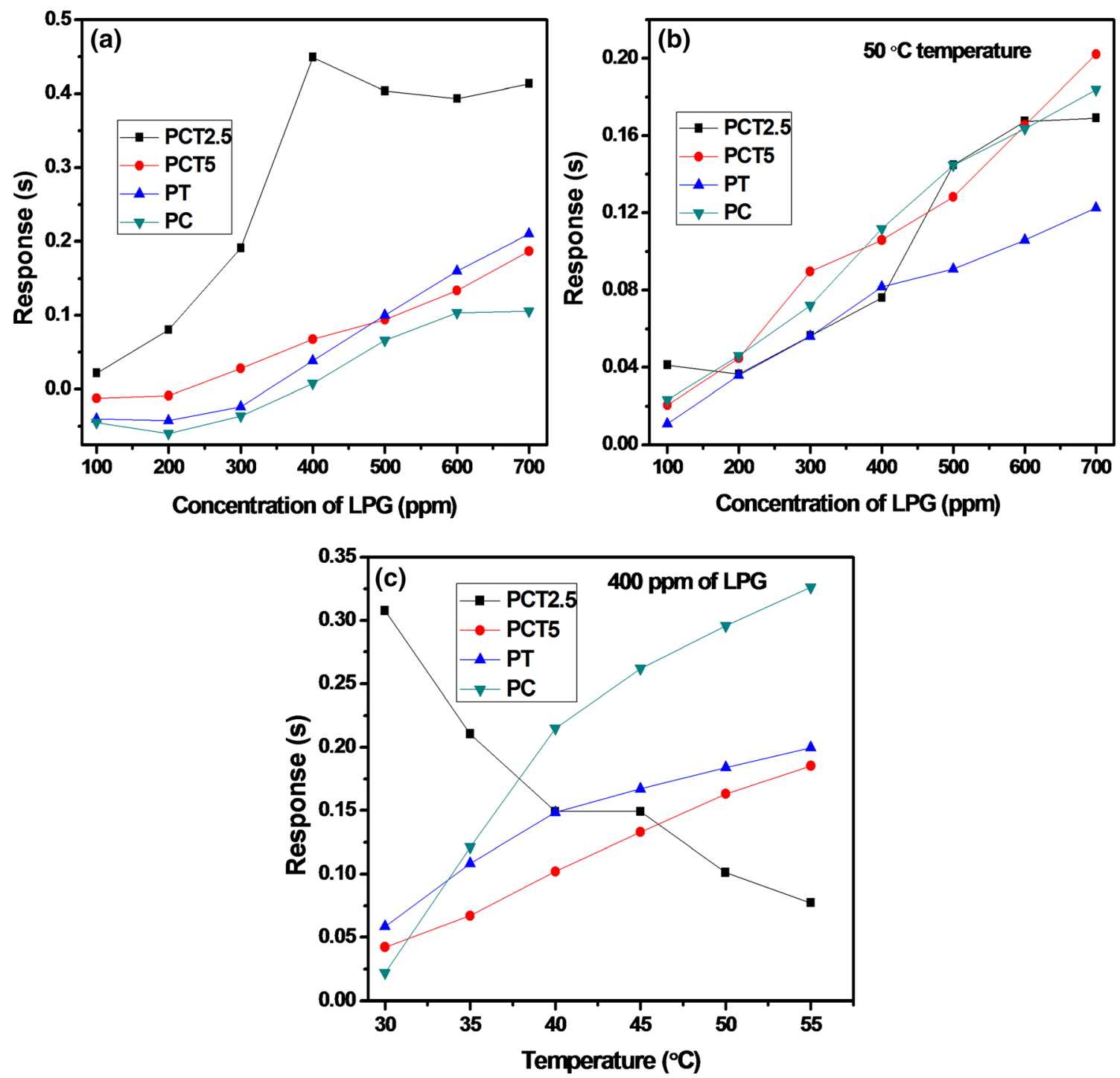

Fig. 2. Sensing response towards LPG at various concentrations (a) at room temperature, (b) at $50^{\circ} \mathrm{C}$ temperature and (c) sensing responses for 400 ppm LPG with temperature variations for all composite samples.

chloroform, ethanol and $\mathrm{SO}_{2}$. In the case of hybrid composite, the interaction between the analyte molecules and the composite surface was higher compared to the only $\mathrm{TiO}_{2}$ filled or CNT filled PVDF. This causes the sensing responses to be lower than that of the hybrid CTNT filled PVDF. The selective LPG sensing is attributed to the good diffusivity of the analyte, better nanocomposite analyte interaction and the charge transfer reaction between the target molecules ${ }^{36,37}$ (all due to better filler dispersibility and network formation). The reusability and long term stability of the sample were also investigated by recording the sensing responses as relative resistance variations over a period of 15 days especially for the LPG gas at 400 ppm concentration. This is shown in Fig. $4 \mathrm{~b}$. The uniform response of the PVDF/CTNT2.5 during the analysis of about 30 days confirms the ability of using this tailored nanocomposite in long-term gas sensing applications.

Figure 5a illustrates the piezoelectric voltage variations observed in the case of PVDF and its composite samples. The corona poled films of the samples exhibited comparatively lower piezoelectric output voltage, attributed to the presence of conducting fillers, and thus the electron transfer reactions taking place within the sample. ${ }^{38}$ Though the neat PVDF and its composites containing $\mathrm{TiO}_{2}$ nanotubes at 2.5 wt. $\%, \mathrm{CNT} / \mathrm{TiO}_{2}$ nanotubes hybrid at $1 \mathrm{wt} . \%$ and $2.5 \mathrm{wt} . \%$ showed output voltage variations when a compressive force of $2.5 \mathrm{~N}$ is imparted on them. The samples with higher content of hybrid nanoparticles and the one with CNT did not show any voltage output. This can be due to the highly conducting nature of the nanocomposites. ${ }^{39}$ Among neat PVDF, PT, PCT1 and PCT2.5, the 

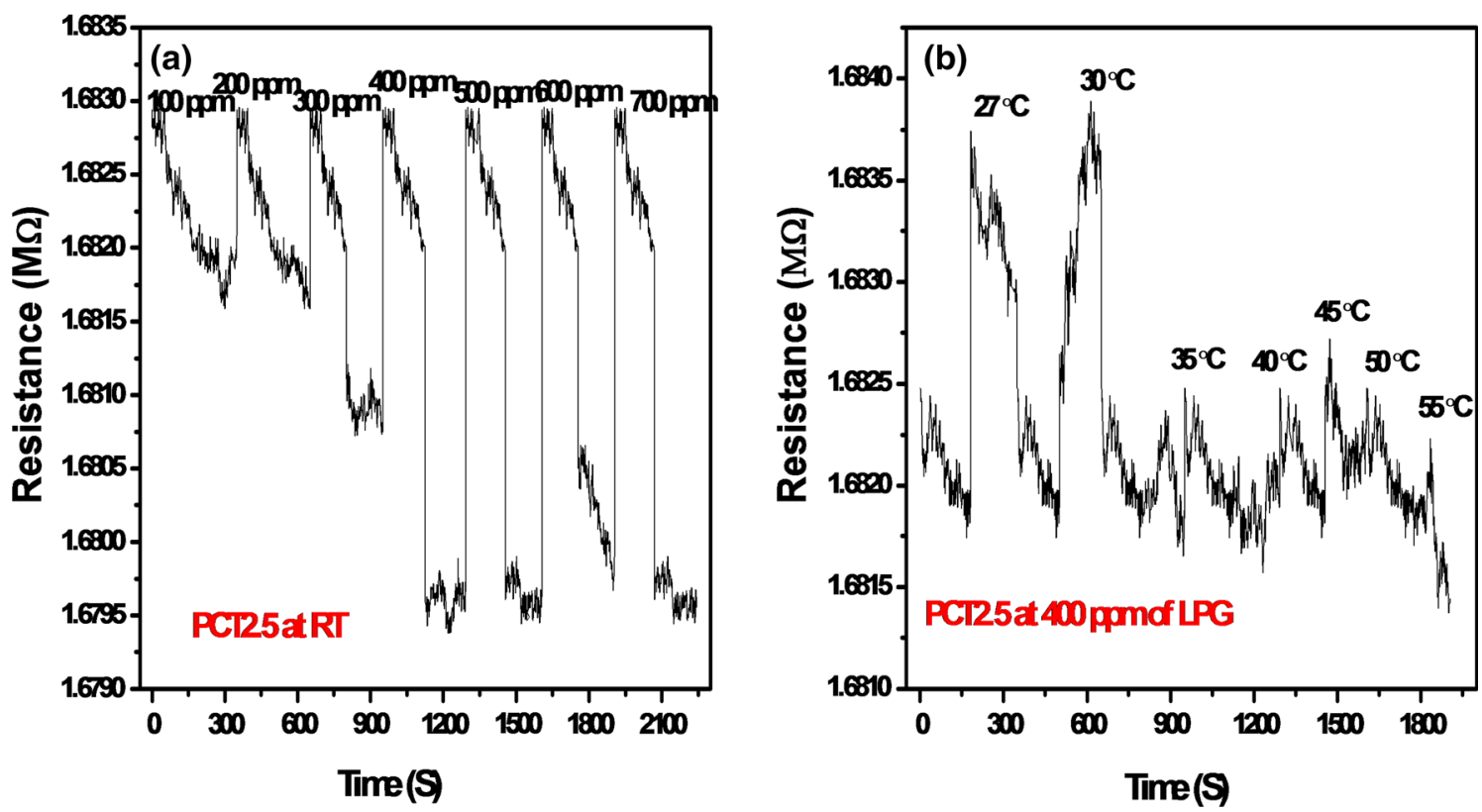

Fig. 3. Dynamic sensor response of the PCT2.5 sample (a) at room temperature for different LPG concentrations and (b) at various temperatures towards the 400 ppm LPG.
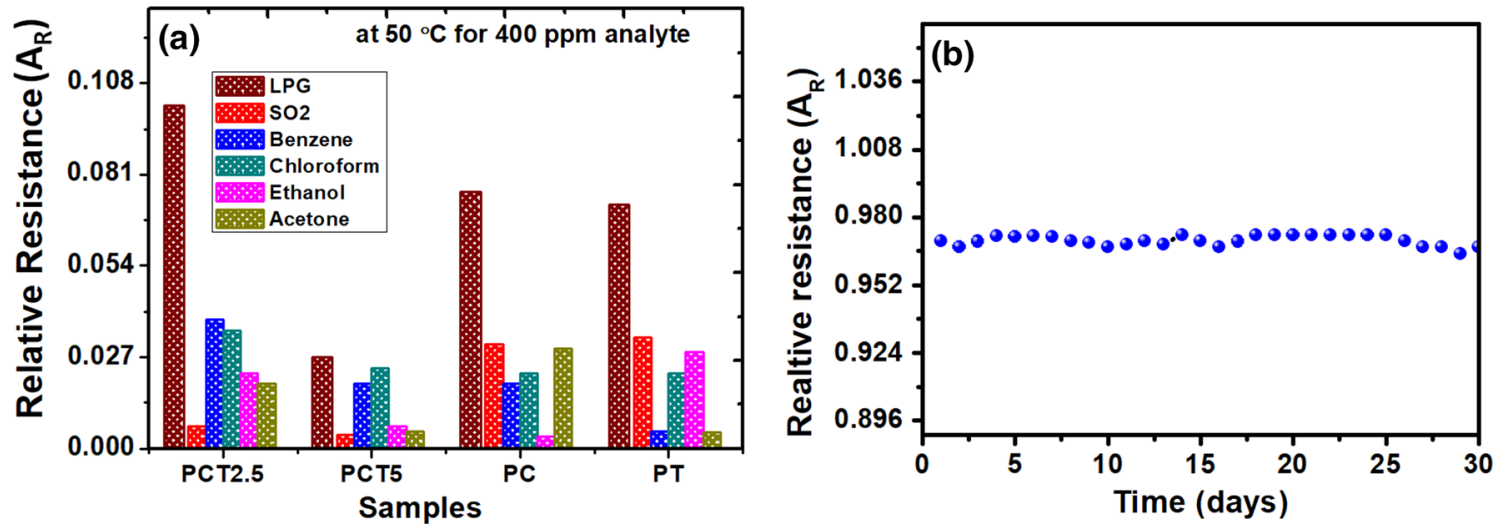

Fig. 4. Solvent vapor sensing performance by the (a) samples and (b) long term stability results for PCT2.5 towards 400 ppm LPG.

PCT1 composite showed the highest piezoelectric output voltage of $1.3 \mathrm{~V}$. This is attributed to the uniform distribution of hybrid ${ }^{40}$ nanotubes within the PVDF medium and the ability of the nanotubes to impart good $\beta$-phase crystallinity to the base polymer. ${ }^{41}$ Influence of nanoparticles on the crystallinity of polymers and their piezoelectric voltage generation was well investigated by our group. The corona poled samples of PVDF copolymer (PVDFHFP) containing barium titanate/hexagonal boron nitride $(3 \mathrm{wt} . \% / 1 \mathrm{wt} . \%)^{18}$ and graphene oxide-titania/strontium titanate $(1: 2)^{22}$ respectively showed maximum output voltages of $2.4 \mathrm{~V}$ and $2.0 \mathrm{~V}$, both being higher than the current performance. This is due to the piezoelectric nature of the filler particles used in the reported works and the larger filler concentration.

Though the best output voltage was observed for the PCT1 sample, best LPG sensing was observed for the PCT2.5 and so this particular sample is selected to test the variations in maximum output voltage during exposure to the gas molecules. The results are shown in Fig. 5b. The PCT2.5 showed a maximum piezoelectric output voltage of $0.8 \mathrm{~V}$ which is observed to decrease during the exposure to LPG. The exposure to LPG was done at regular intervals on the same sample between which $\mathrm{N}_{2}$ gas was passed through the sample to achieve neutral nature. During the LPG exposure at $400 \mathrm{ppm}$ the voltage changes to $0.3 \mathrm{~V}$. This voltage variation is pronounced when compared to the similar output piezo voltage $(0.533 \mathrm{~V}$ in air shifts to $0.089 \mathrm{~V} \mathrm{LPG})$ variations observed for $\mathrm{ZnSnO}_{3} / \mathrm{ZnO}$ nanowires towards $8000 \mathrm{ppm}$ LPG. ${ }^{42}$ As illustrated in the sensing characteristics, the sample exposure to LPG generates electrons within and such electrons move towards the conduction band. This cases the carrier density of the nanotubes to be enhanced and 

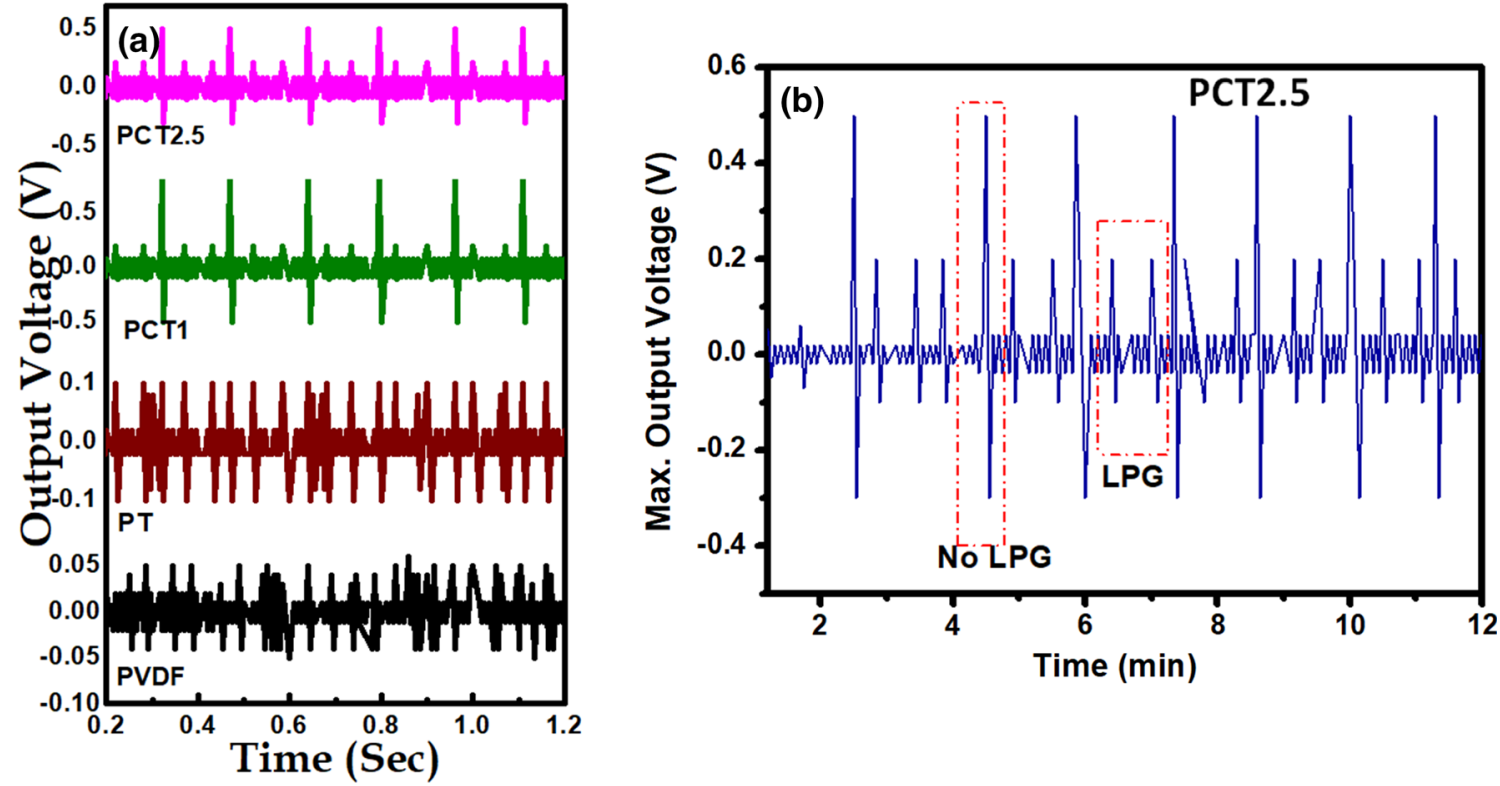

Fig. 5. (a) Piezoelectric output voltage obtained for the samples, neat polymer, PT, PC, PCT1 and PCT2.5 composites and (b) maximum output voltage variations when the PCT2.5 sample is exposed to and recovered from 400 ppm LPG.

decreases the surface depletion layer width. ${ }^{43}$ The compressive force applied during the piezoelectric experiment enhances the charge carrier density and causes piezoelectric screening effect. This finally results in the decreased output voltage. ${ }^{43-45}$

Morphology and structural investigations of the synthesized hybrid nanotubes are well demonstrated in our previously published research. ${ }^{24}$ The hydrothermal synthesis of $\mathrm{TiO}_{2}$ nanotubes and the $\mathrm{CNT}_{-} \mathrm{TiO}_{2}$ nano hybrid material exhibited tubular morphology. The dense, interconnected and coiled network of tubes was seen in the hybrid CNT$\mathrm{TiO}_{2}$. The tube-in-tube and tube-on-tube structure as $\mathrm{TiO}_{2}$ tubes grow on the walls and inner surfaces of the CNTs were observed. No rupture or formation of chemical bonds was seen in the case of CNT-TiO hybrid nanomaterial, indicating only physical/electronic interactions between both tubes. ${ }^{24}$ Morphology images for the cryocut surfaces of PVDF nanocomposites are provided in Fig. 6.

The planar morphology of the PVDF neat polymer was transformed when specific nanomaterials are incorporated in it. The projections observed in Fig. 6a, b, c, and d indicates the tubular CNT and $\mathrm{TiO}_{2}$ nanomaterials. Better uniformity in dispersion of nanotubes was observed in Fig. $6 \mathrm{~b}$ and c, respectively, where CNT and CNT-TiO 2 were present at 2.5 wt.\%. This indicates the ability of CNTs in uniformly distributing the $\mathrm{TiO}_{2}$ tubes, as they were grown within the CNT structures. ${ }^{24}$ This result confirms the hybrid networking effect of nanomaterials in uniformly distributing themselves within the polymer matrix. However, at 5 wt.\% (Fig. 6d), the higher concentrations result in uniformity lose in dispersion and thus agglomerated structural deformations are observed. These results are in good agreement with the sensing responses obtained for all samples, and the piezoelectric property enhancement.

Structural investigations on the PVDF nanocomposites were done using the FTIR and XRD studies. As shown in Fig. 7a, the FTIR spectra for all samples are similar as the pronounced peaks are due to the $\mathrm{C}-\mathrm{C}$ stretching, $\mathrm{C}-\mathrm{H}$ stretching and bending vibrations of the PVDF skeleton. ${ }^{20,28}$ No significant variation in the peak positions, or peak intensities confirm the absence of any kind of chemical interactions that the nanomaterials impart on the polymer chains. The FTIR spectra shown in Fig. 7a does not provide any information for chemical grafting between the polymer and the nanofillers. This also indicates the weak physical interactions between the filler and PVDF. ${ }^{18}$ XRD patterns of the samples are shown in Fig. $7 \mathrm{~b}$ with peaks oriented at $17.5^{\circ}, 18.5^{\circ}, 20^{\circ}$ and $26.5^{\circ}$ attributed to the crystalline phases of PVDF. It is known that the peaks at $18.5^{\circ}, 20^{\circ}$ and $26.5^{\circ}$ correspond to the crystalline alpha phase, with similar peak around $20^{\circ}$ corresponding to the beta phase. The large intensity of the peak around $20^{\circ}$ is attributed to the combined influence of the alpha and beta crystalline phases. There is a slight enhancement in the intensity of XRD peaks indicating the effect of tubular filler materials on the crystallinity values of PVDF. ${ }^{8,18,20-22}$ This enhancement in crystallinity is the main factor behind the higher piezoelectric output voltage obtained for the sample containing CNT and $\mathrm{TiO}_{2}$ nanotubes.

The surface characteristics of the nanocomposites are explored by means of contact angle measurements as provided in Table I. The contact angle values show good variation between various 

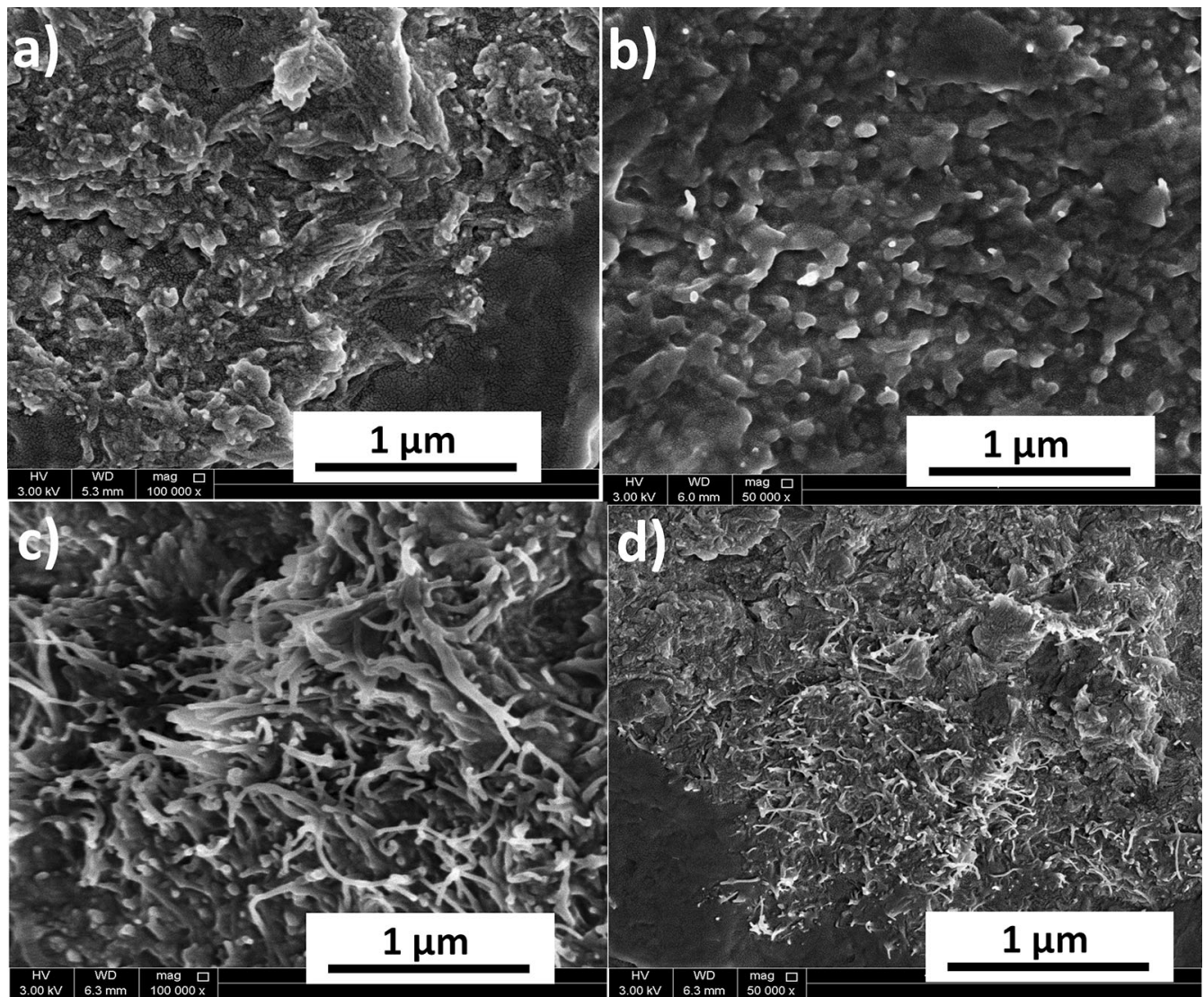

Fig. 6. Scanning electron microscopy images for (a) PT, (b) PC, (c) PCT2.5 and (d) PCT5 nanocomposites.
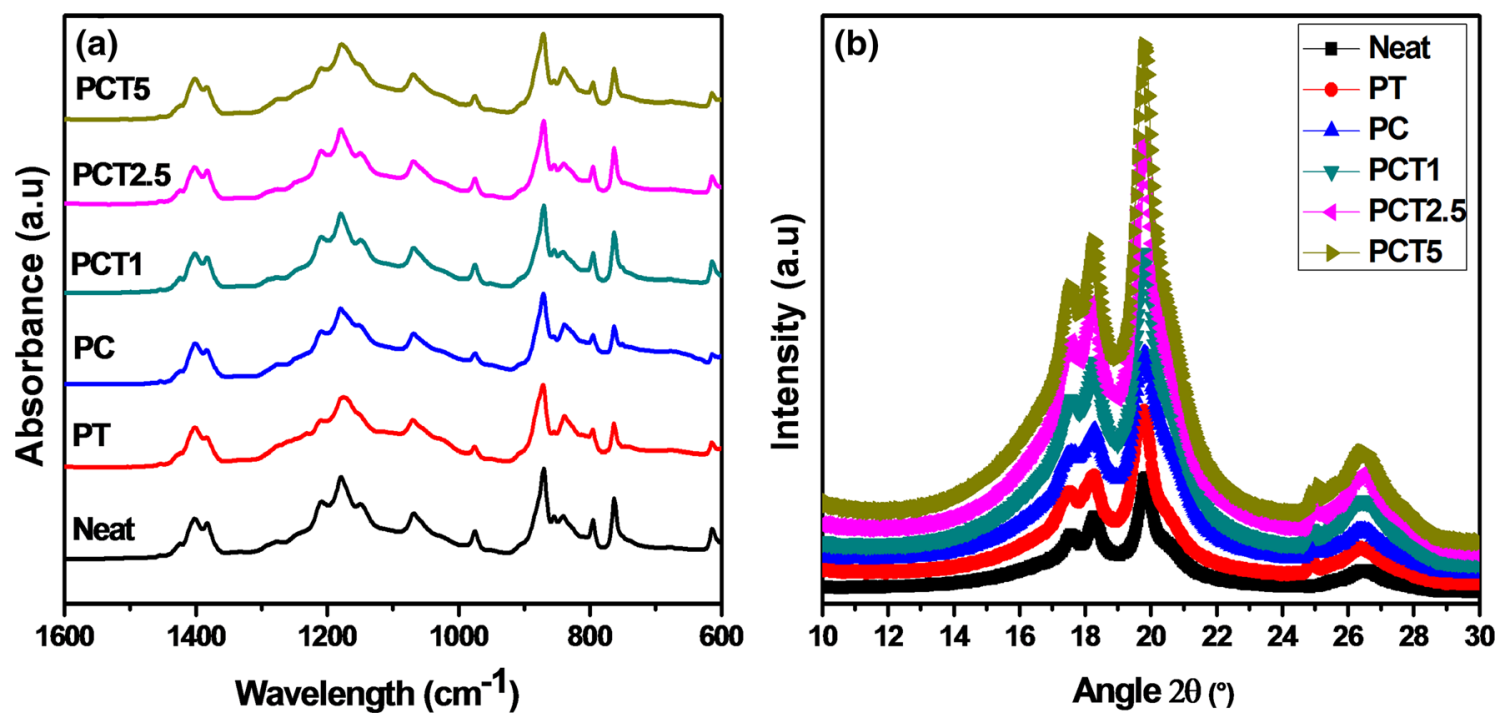

Fig. 7. (a) FTIR spectra and (b) x-ray diffraction patterns for the PVDF/CTNT composites.

samples, according to the nature of the nanotubes. It is clear that both nanotubes enhance the hydrophobicity of PVDF, however, at $2.5 \mathrm{wt} . \%$, all nanocomposites show similar contact angle values. This can be due to the better dispersion of nanotubes in correlation with the SEM results.
The melting and crystallization curves for the PVDF nanocomposites are represented in Fig. 8. The crystallization peaks (Fig. 8a) are sharp indicating the uniform crystalline structure of the composites. The crystallization kinetics indicates the formation of crystallites at higher temperature. When the values are compared there is a slight 
change in the crystallization temperature indicating the stability of the nanocomposites. ${ }^{22}$ This is a good sign as far as the piezoelectric property enhancement is considered. The fillers have the ability to enhance the crystallinity of the PVDF, and this in turn influence the piezoelectric output voltage.

The increase in concentration of the filler particles and the mode of dispersion and interfacial stability achieved by the presence of hybrid nanotubes have pronounced effect on the variation in these characteristic parameters. In Fig. $8 \mathrm{~b}$ also, the melting curves show slight change with the addition of filler particles. This indicates the formation of uniform filler networks within the polymer and the enhanced thermal stability and glass transition for the PVDF nanocomposites containing $\mathrm{CNT}^{-\mathrm{TiO}_{2}}$ hybrid nanotubes. ${ }^{24}$

The ability to store electric charge in a material is indicated by its dielectric properties, and it is measured in terms of dielectric constant, dielectric loss and conductivity values as shown in Fig. 9. The dielectric constant values enhanced with the nanotubes addition as clear from Fig. 9a. This dielectric permittivity enhancement with the presence of filler particles is generally explained in terms of Maxwell-Wagnar-Sillars effect, ${ }^{10,13}$ which explains the

Table I. Contact angle measurements for the PVDF composites

\begin{tabular}{lc}
\hline Sample & Contact angle \\
\cline { 1 - 1 } & $90.83 \pm 1.87$ \\
PT & $91.34 \pm 3.34$ \\
PC & $92.40 \pm 1.92$ \\
PCT1 & $90.28 \pm 3.54$ \\
PCT2.5 & $91.78 \pm 3.02$ \\
PCT5 & $93.07 \pm 1.52$ \\
\hline
\end{tabular}

significance of heterogeneous interfaces (filler-polymer) in regulating electron transfer mechanism. Lower amounts of the particles and the less dispersion rates do not cause much filler-filler interactions and network formation within the polymer nanocomposites, causing many defects and voids in the interfacial junctions. However, increased filler percentages connects throughout and make a conductive network, this again supports the good sensing response observed for the PCT2.5 sample. The conductivity values showed in Fig. 9b explains the significance of CNT networks in imparting better electron transfer and movement as the CNT containing samples shows relatively higher conductivity. The $\mathrm{TiO}_{2}$ nanotubes grown on CNT also improves the conductivity through better dispersion and network formation. Thus, the dielectric results are also in correlation with the sensing, piezoelectric and morphology data.

\section{CONCLUSIONS}

This paper describes the fabrication of PVDFbased composite films containing CNT-TiO $\mathrm{C}_{2}$ hybrid nanotube architecture for their effective application in LPG sensing. The hybrid architecture for the nanotubes was obtained by the hydrothermal method and solution casted sample films were used for conventional characteristic property assessment. The sensor responses depend on the rupture and formation of the conducting network within the samples, and this is ensured by the well dispersed hybrid CNT- $\mathrm{TiO}_{2}$ network. The piezoelectric output voltage varies from $0.8 \mathrm{~V}$ to $0.3 \mathrm{~V}$ with the exposure to LPG. The dielectric permittivity and tan delta values for the composites also followed the similar explanations of electron transfer and conductive filler particles-based mechanism. Variation in piezoelectric output voltage of the PCT2.5 sample shows the applicability of this nanocomposite in fabricating self-powering gas sensors.
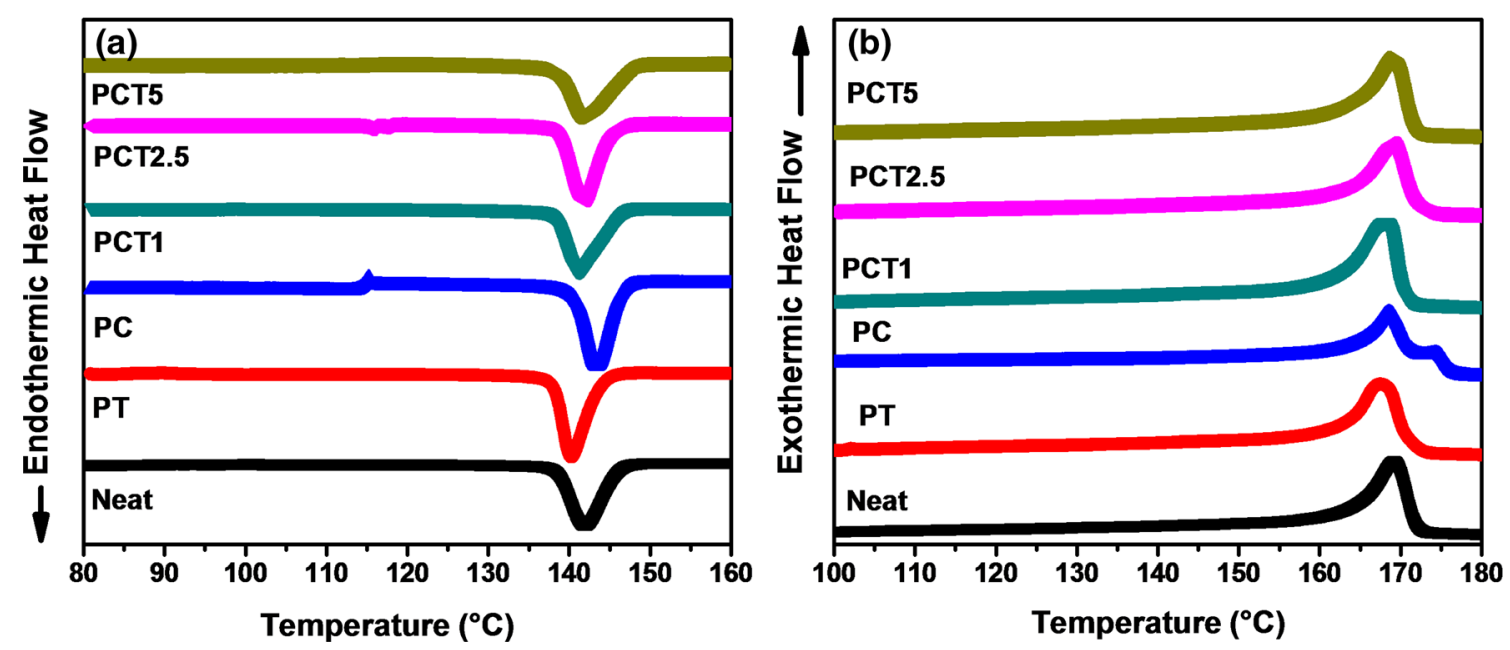

Fig. 8. (a) Melting and (b) crystallization curves of PVDF nanocomposites containing CNT, $\mathrm{TiO}_{2}$ and $\mathrm{CNT}^{-\mathrm{TiO}_{2}}$ hybrid nanotubes. 

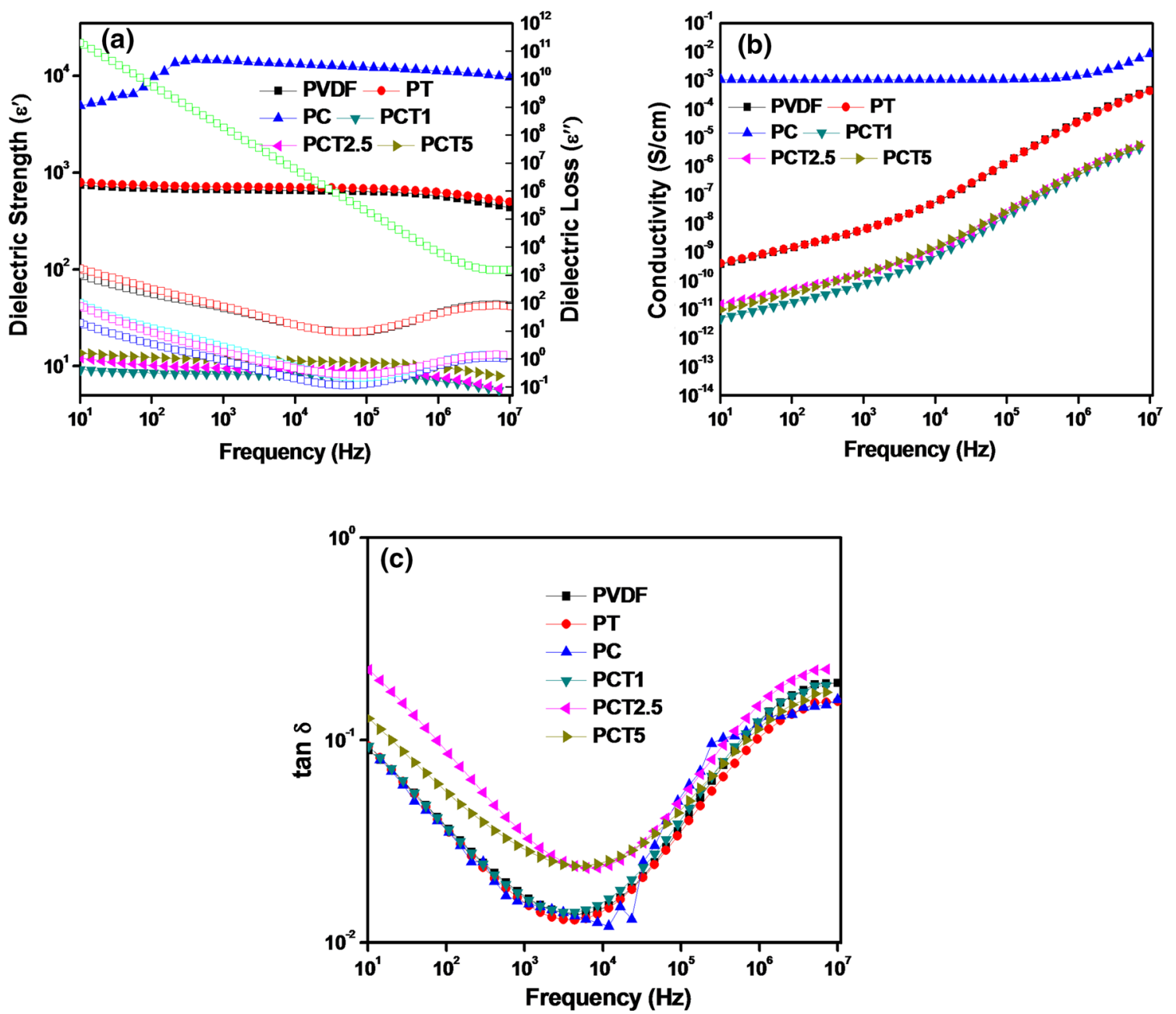

Fig. 9. Dielectric data for PVDF/CTNT composites versus frequency (a) dielectric strength and dielectric loss, (b) conductivity and (c) tan $\delta$.

\section{ACKNOWLEDGMENTS}

Open Access funding provided by the Qatar National Library. This publication was made possible by NPRP Grant 6-282-2-119 from the Qatar National Research Fund (a member of Qatar Foundation). The statements made herein are solely the responsibility of the authors.

\section{CONFLICT OF INTEREST}

The authors declare that they have no conflict of interest.

\section{OPEN ACCESS}

This article is licensed under a Creative Commons Attribution 4.0 International License, which permits use, sharing, adaptation, distribution and reproduction in any medium or format, as long as you give appropriate credit to the original author(s) and the source, provide a link to the Creative Commons licence, and indicate if changes were made. The images or other third party material in this article are included in the article's Creative Commons li- cence, unless indicated otherwise in a credit line to the material. If material is not included in the article's Creative Commons licence and your intended use is not permitted by statutory regulation or exceeds the permitted use, you will need to obtain permission directly from the copyright holder. To view a copy of this licence, visit http://creativecommons.org/licenses/by/4.0/.

\section{REFERENCES}

1. D. Ponnamma, K.K. Sadasivuni, C. Wan, S. Thomas, and M.A. AlMa'adeed, eds., Flexible and Stretchable Electronic Composites (Berlin: Springer, 2015).

2. G. Georgousis, C. Pandis, A. Kalamiotis, P. Georgiopoulos, A. Kyritsis, E. Kontou, P. Pissis, M. Micusik, K. Czanikova, J. Kulicek, and M. Omastova, Compos. B Eng. 68, 162 (2015).

3. K.K. Sadasivuni, D. Ponnamma, J. Kim, and S. Thomas, eds., Graphene-Based Polymer Nanocomposites in Electronics (Cham: Springer, 2015).

4. V. Eswaraiah, K. Balasubramaniam, and S. Ramaprabhu, J. Mater. Chem. 21, 12626 (2011).

5. D. Ponnamma, Q. Guo, I. Krupa, M.A. Al-Maadeed, K.T. Varughese, S. Thomas, and K.K. Sadasivuni, Phys. Chem. Chem. Phys. 17, 3954 (2015). 
6. D. Ponnamma, A. Saiter, J.M. Saiter, S. Thomas, Y. Grohens, M.A. AlMaadeed, and K.K. Sadasivuni, J. Polym. Res. 23, 125 (2016).

7. D. Ponnamma, K.T. Varughese, M.A. Al-Maadeed, and S. Thomas, Polym. Int. 66, 931 (2017).

8. A. Al-Saygh, D. Ponnamma, M.A. AlMaadeed, P. Vijayan, A. Karim, and M. Hassan, Polymers 9, 33 (2017).

9. A. Ferrreira, J.G. Rocha, A. Ansón-Casaos, M.T. Martínez, F. Vaz, and S. Lanceros-Mendez, Sens. Actuators A Phys. 178,10 (2012).

10. J.G. Thangamani, K. Deshmukh, K.K. Sadasivuni, D. Ponnamma, S. Goutham, K.V. Rao, K. Chidambaram, M.B. Ahamed, A.N. Grace, M. Faisal, and S.K. Pasha, Microchim. Acta 184, 3977 (2017).

11. K. Ke, P. Pötschke, N. Wiegand, B. Krause, B. Voit, and A.C.S. Appl, Mater. Interfaces 8, 14190 (2016).

12. Z. Zhu, L. Garcia-Gancedo, A.J. Flewitt, H. Xie, F. Moussy, and W.I. Milne, Sensors 12, 5996 (2012).

13. N. Raphael, K. Namratha, B.N. Chandrashekar, K.K. Sadasivuni, D. Ponnamma, A.S. Smitha, S. Krishnaveni, C. Cheng, and K. Byrappa, Prog. Cryst. Growth Charact. Mater. 64, 75 (2018).

14. D. Ponnamma, S.H. Sung, J.S. Hong, K.H. Ahn, K.T. Varughese, and S. Thomas, Eur. Polym. J. 53, 147 (2014).

15. M. Wang, K. Zhang, X.X. Dai, Y. Li, J. Guo, H. Liu, G.H. Li, Y.J. Tan, J.B. Zeng, and Z. Guo, Nanoscale 9, 11017 (2017).

16. S.M. Hosseini and A.A. Yousefi, Org. Electron. 50, 121 (2017).

17. K. Zhang, J. Ji, X. Fang, L. Yan, and B. Liu, Analyst 140, 134 (2015).

18. D. Ponnamma and M. Al-Maadeed, Sustain. Energy Fuel 3, 774 (2019).

19. D. Ponnamma, S. Goutham, K.K. Sadasivuni, K.V. Rao, J.J. Cabibihan, and M.A. Al-Maadeed, Synth. Metals 243, 34 (2018).

20. H. Parangusan, D. Ponnamma, and M.A. AlMaadeed, Soft Matter 14, 8803 (2018).

21. A. Issa, M.A. Al-Maadeed, A. Luyt, D. Ponnamma, and M. Hassan, Carbon Mater. 3, 30 (2017).

22. D. Ponnamma, A. Erturk, H. Parangusan, K. Deshmukh, M.B. Ahamed, and M.A. Al-Maadeed, Emerg. Mater. 1, 55 (2018).

23. C. Hu, Z. Li, Y. Wang, J. Gao, K. Dai, G. Zheng, C. Liu, C. Shen, H. Song, and Z. Guo, J. Mater. Chem. C 5, 2318 (2017).

24. M.M. Chamakh, D. Ponnamma, and M.A. Al-Maadeed, J. Mater. Sci. Mater. Electron. 29, 4402 (2018).
25. D. Ponnamma and M.A. Al-Maadeed, Mater. Des. 117, 203 (2017).

26. S. Goutham, S. Bykkam, K.K. Sadasivuni, D.S. Kumar, M. Ahmadipour, Z.A. Ahmad, and K.V. Rao, Microchim. Acta 185, 69 (2018).

27. J.M. Park, G.Y. Gu, Z.J. Wang, D.J. Kwon, and K.L. DeVries, Appl. Surf. Sci. 287, 75 (2013).

28. H. Parangusan, D. Ponnamma, and M.A. AlMaadeed, RSC Adv. 7, 50156 (2017).

29. M. Knite, V. Tupureina, A. Fuith, J. Zavickis, and V. Teteris, Mater. Sci. Eng. C 27, 1125 (2007).

30. R. Thirupathi, G. Solleti, T. Sreekanth, K.K. Sadasivuni, and K.V. Rao, J. Electron. Mater. 47, 3468 (2018).

31. W.L. Ong, M. Gao, and G.W. Ho, Nanoscale. 5, 11283 (2013).

32. R.K. Sonker, M. Singh, U. Kumar, and B.C. Yadav, J. Inorg. Organomet. Polym. Mater. 26, 1434 (2016).

33. T. Sen, N.G. Shimpi, S. Mishra, and R. Sharma, Sens. Actuators B 190, 120 (2014).

34. A. Parveen, A. Koppalkar, and A.S. Roy, Sens. Lett. 11, 242 (2013).

35. A. Kaushik, R. Kumar, S.K. Arya, M. Nair, B.D. Malhotra, and S. Bhansali, Chem. Rev. 115, 4571 (2015).

36. R.K. Mishra, S.B. Upadhyay, A. Kushwaha, T.H. Kim, G. Murali, R. Verma, M. Srivastava, J. Singh, P.P. Sahay, and S.H. Lee, Nanoscale 7, 11971 (2015).

37. A. Wei, L. Pan, and W. Huang, Mater. Sci. Eng. B 176, 1409 (2011)

38. F. Yang, Y.M. Wen, P. Li, M. Zheng, and L.X. Bian, Sens. Actuators A Phys. 141, 129 (2008).

39. H. Zhao, J. Bai, and A.C.S. Appl, Mater. Interfaces 7, 9652 (2015).

40. W. Brostow, S. Lohse, X. Lu, and A.T. Osmanson, Emerg. Mater. 2, 23 (2018)

41. Y. Ahn, J.Y. Lim, S.M. Hong, J. Lee, J. Ha, H.J. Choi, and Y. Seo, J. Phys. Chem. C 117, 11791 (2013).

42. Y. Fu, Y. Nie, Y. Zhao, P. Wang, L. Xing, Y. Zhang, and X. Xue, ACS Appl. Mater. Interfaces 7, 10482 (2015).

43. P. Wang, Y. Fu, B. Yu, Y. Zhao, L. Xing, and X. Xue, J. Mater. Chem. A 3, 3529 (2015).

44. Z. Qu, Y. Fu, B. Yu, P. Deng, L. Xing, and X. Xue, Sens. Actuators B Chem. 222, 78 (2016).

45. H. He, Y. Fu, W. Zang, Q. Wang, L. Xing, Y. Zhang, and X. Xue, Nano Energy 31, 37 (2017).

Publisher's Note Springer Nature remains neutral with regard to jurisdictional claims in published maps and institutional affiliations. 